But biotech companies start with a clean piece of paper. There's more flexibility and more original thinking in putting deals together," says Wiltsey.

\section{Covering up weak pipelines}

Pharmaceutical companies will increasingly turn to biotech companies to cover pipeline deficiencies, according to David Hale, president and CEO of Gensia Pharmaceuticals (San Diego, CA). Most pharmaceutical-industry analysts expect pharmaceutical companies to achieve annual growth rates of 10 percent to 15 percent. This means a pharmaceutical company with annual sales of $\$ 3$ billion must generate $\$ 450$ million in new sales next year and $\$ 520$ million in such sales the year after. "The only way to do this is by introducing newdrugs- those drugs that biotechnology is developing," Hale says. While Glaxo (London), Pfizer (New York), and Merck (Rahway, NJ) have sufficiently strong pipelines, most pharmaceutical companies have "terrible pipelines that won't lead to significant products for some time," says Hale

Previously, pharmaceutical companies offset a lack of new products with price increases on old ones. But continuing federal-government pressure on pharmaceutical prices will limit future increases. So pharmaceutical companies will have little choice but to use their "huge cash reserves as strategic assests. Biotech companies will thus have increasing opportunities to show pharmaceutical companies creative ways to use their assests to gain access to innovative drugs," Hale says.

Chiron's Penhoet sounded the panel's lone note of humility as the luncheon drew to a close. "We don't want to give the impression of discounting pharmaceutical companies-they're tough competition," Penhoet said. "Moving drugs through development, as well as marketing and distribution, are the pharmaceutical-company strengths."Conspicuously, Penhoet didn't list research as a pharmaceutical-company asset. -B.J.Spalding

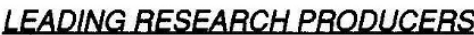 ACADEMIC}

NEW YORK - Counting published research papers is a rough gauge of a laboratory's output. The Institute for Scientific Information (ISI, Philadelphia, PA), however, not only counts published papers, it determines how many citations the papers received and the average number of citations per paper.

By these measures, 15 surveyed independent labs and university labs outperformed 10 surveyed biotechnology companies. Both the labs and biotech companies, moreover, far outperformed 10 surveyed pharmaceutical companies.

An ISI analyst puts these findings in perspective. "The attitude toward publishing is paramount. In academia, publishing is a necessity. Since biotechnology companies were started by academics, the culture has carried over. But pharmaceutical companies place less emphasis on publishing. Some of their best work isn't published," says David Pendlebury, editor of ISI's Science Watch.

The 15 independent labs and university labs clearly dominated, as their papers published between January of 1981 and June of 1992 earned an average of 31.3 citations each (Table 1). The Whitehead Institute (Cambridge, MA) led the way, with 51 citations per paper, on average. Massachusetts Institute of Technology's (Cambridge, MA) Department of Biology was runner up, with an average of 46.8 citations per paper.

The 10 biotech companies-with an average of 27.5 citations per paperweren't far behind. Genentech (S. San Francisco, CA) was the front runner, with 39.6 citations per paper, on average, while Genetics Institute (Cambridge, $\mathrm{MA}$ ) followed, with an average of 37.5 citations per paper. Genentech-which funded the ISI study-performed particularlywell. Fully 1 percent of its papers were cited over 500 times, whereas, typically, only 0.03 percent of papers are

\title{
RESEARCH IS BETTER
}

cited that often. "Genentech produces a lot of blockbuster papers," says Pendlebury.

The 10 pharmaceutical companies, for their part, brought up the rear, with an average of 10.8 citations per paper.John- son \& Johnson (New Brunswick, NJ) was the leader, as its papers earned 21 citations each. Merck (Rahway, NJ) placed second, with its papers capturing an average of 17 citations apiece.

-B.J. Spalding

\begin{tabular}{|c|c|c|c|}
\hline Independent and University Laboratories & Papers* & Citations $^{*}$ & Citations Per Paper \\
\hline $\begin{array}{l}\text { Whitehead Institute } \\
\text { Massachusetts Institute of Technology } \\
\text { Department of Biology }\end{array}$ & $\begin{array}{l}1,035 \\
3,139\end{array}$ & $\begin{array}{r}52,820 \\
146,770\end{array}$ & $\begin{array}{l}51.03 \\
46.76\end{array}$ \\
\hline $\begin{array}{l}\text { Carnegie Institution } \\
\text { Department of Embryology }\end{array}$ & 408 & 18,917 & 46.37 \\
\hline $\begin{array}{l}\text { Cold Spring Harbor } \\
\text { Salk Institute }\end{array}$ & $\begin{array}{l}1,139 \\
3,612\end{array}$ & $\begin{array}{r}51,926 \\
144,739\end{array}$ & $\begin{array}{l}45.59 \\
40.07\end{array}$ \\
\hline $\begin{array}{l}\text { University of California (San Francisco) } \\
\text { Department of Biochemistry \& Biophysics }\end{array}$ & 2,537 & 85,826 & 33.83 \\
\hline $\begin{array}{l}\text { La Jolla Cancer Research Center } \\
\text { Hutchinson Cancer Center }\end{array}$ & $\begin{array}{l}1,037 \\
3,562\end{array}$ & $\begin{array}{l}28,424 \\
94,585\end{array}$ & $\begin{array}{l}27.41 \\
26.55\end{array}$ \\
\hline $\begin{array}{l}\text { Stanford University } \\
\text { Department of Genetics }\end{array}$ & 741 & 19,207 & 25.92 \\
\hline $\begin{array}{l}\text { University of California (Berkeley) } \\
\text { Division of Cell \& Molecular Biology }\end{array}$ & 4,252 & 99,233 & 23.34 \\
\hline $\begin{array}{l}\text { Wistar Institute } \\
\text { National Institutes of Health (NIH) }\end{array}$ & $\begin{array}{r}2,611 \\
35,462\end{array}$ & $\begin{array}{r}59,028 \\
782,595\end{array}$ & $\begin{array}{l}22.61 \\
22.07\end{array}$ \\
\hline $\begin{array}{l}\text { Princeton University } \\
\text { Department of Biology }\end{array}$ & 1,201 & 24,576 & 20.46 \\
\hline $\begin{array}{l}\text { National Jewish Center for Immunology } \\
\text { \& Respiratory Medicine }\end{array}$ & 2,636 & 49,560 & 18.80 \\
\hline $\begin{array}{l}\text { Scripps } \\
\text { Average }\end{array}$ & $\begin{array}{l}7,815 \\
4,746\end{array}$ & $\begin{array}{l}140,525 \\
119,915\end{array}$ & $\begin{array}{l}17.98 \\
31.25\end{array}$ \\
\hline \multicolumn{4}{|l|}{ Biotechnology Companies_ } \\
\hline $\begin{array}{l}\text { Genentech } \\
\text { Genetics Institute } \\
\text { Biogen } \\
\text { Chiron } \\
\text { Centocor } \\
\text { Immunex } \\
\text { Cambridge Biotech } \\
\text { Scios Nova } \\
\text { Amgen } \\
\text { Genzyme } \\
\text { Average }\end{array}$ & $\begin{array}{r}2,181 \\
553 \\
568 \\
1,691 \\
243 \\
541 \\
140 \\
533 \\
570 \\
77 \\
710\end{array}$ & $\begin{array}{r}86,258 \\
20,759 \\
20,258 \\
55,493 \\
6,787 \\
14,858 \\
3,660 \\
10,190 \\
9,983 \\
831 \\
22,908\end{array}$ & $\begin{array}{l}39.55 \\
37.54 \\
35.67 \\
32.82 \\
27.93 \\
27.46 \\
26.14 \\
19.12 \\
17.51 \\
10.79 \\
27.50\end{array}$ \\
\hline \multicolumn{4}{|l|}{ Pharmaceutical Companies } \\
\hline $\begin{array}{l}\text { Johnson \& Johnson } \\
\text { Merck } \\
\text { Roche Group } \\
\text { Eli Lilly } \\
\text { Glaxo Holdings } \\
\text { Pfizer } \\
\text { Bristol-Myers Squibb } \\
\text { Abbott Labs } \\
\text { American Home } \\
\text { SmithKline Beecham } \\
\text { Average }\end{array}$ & $\begin{array}{r}452 \\
3,690 \\
3,764 \\
2,061 \\
1,232 \\
826 \\
1,638 \\
1,423 \\
882 \\
2,016 \\
1,798\end{array}$ & $\begin{array}{r}9,480 \\
62,893 \\
62,047 \\
22,452 \\
11,338 \\
7,291 \\
11,904 \\
9,601 \\
4,964 \\
10,461 \\
\mathbf{2 1 , 2 4 3}\end{array}$ & $\begin{array}{l}20.97 \\
17.04 \\
16.48 \\
10.89 \\
9.20 \\
8.83 \\
7.27 \\
6.75 \\
5.63 \\
5.19 \\
10.8\end{array}$ \\
\hline
\end{tabular}

